\title{
Intrathecal administration of rapamycin inhibits the phosphorylation of DRG Nav1.8 and attenuates STZ-induced painful diabetic neuropathy in rats
}

Wan-you He ${ }^{1}$, Bin Zhang ${ }^{1}$, Qing-ming Xiong, Cheng-xiang Yang, Wei-cheng Zhao, Jian He, Jun Zhou, Han-bing Wang*

Department of Anesthesiology, The First People's Hospital of Foshan, 81\# North of LingNan Road, Foshan 528000, China

\begin{abstract}
The mammalian target of rapamycin (mTOR) is a key regulator of mRNA translation and protein synthesis, and it is specifically inhibited by rapamycin. In chronic pain conditions, mTOR-mediated local protein synthesis is crucial for neuronal hyperexcitability and synaptic plasticity. The tetrodotoxin-resistant (TTX-R) sodium channel Nav1.8 plays a major role in action potential initiation and propagation and cellular excitability in DRG (dorsal root ganglion) neurons. In this study, we investigated if mTOR modulates the phosphorylation of Nav1.8 that is associated with neuronal hyperexcitability and behavioral hypersensitivity in STZ-induced diabetic rats. Painful diabetic neuropathy (PDN) was induced in Sprague-Dawley rats by intraperitoneal injection with streptozotocin (STZ) at 60 $\mathrm{mg} / \mathrm{kg}$. After the onset of PDN, the rats received daily intrathecal administrations of rapamycin $(1 \mu \mathrm{g}, 3 \mu \mathrm{g}$, or $10 \mu \mathrm{g} /$ day) for 7 days; other diabetic rats received the same volumes of dimethyl sulfoxide (DMSO). Herein, we demonstrate a marked increase in protein expression of total mTOR and phospho-mTOR (p-mTOR) together with the up-regulation of phosphor-Nav1.8 (p-Nav1.8) prior to the mechanical withdrawal threshold reaching a significant reduction in dorsal root ganglions (DRGs).

Furthermore, the intrathecal administration of rapamycin, inhibiting the activity of mTOR, suppressed the phosphorylation of DRG Nav1.8, reduced the TTX-R current density, heightened the voltage threshold for activation and lowered the voltage threshold for inactivation and relieved mechanical hypersensitivity in diabetic rats. An intrathecal injection (i.t.) of rapamycin inhibited the phosphorylation and enhanced
\end{abstract}

\footnotetext{
Abbreviations: STZ, streptozotocin; PDN, painful diabetic neuropathy; DRG, dorsal root ganglion; mTOR, Mammalian target of rapamycin; S6K, ribosomal protein S6 kinase; 4E-BP, eukaryotic initiation factor 4E (eIF4E) binding protein; IR, insulin receptor; IRS-1, insulin receptor substrate-1;ERK, extracellular regulated protein kinases; i.t., intrathecal injection; MWT, mechanical withdrawal threshold.

${ }^{*}$ Correspondence: Tel.: +86 757 83162513; fax: +86 75783162513

E-mail address: fswhbing@163.com (H.B. Wang)

${ }^{1}$ These authors contributed equally to this work
} 
the functional availability of DRG Nav1.8 attenuated STZ-induced hyperalgesia. These results suggest that rapamycin is a potential therapeutic intervention for clinical PDN.

\section{Keywords}

mTOR; Painful diabetic neuropathy; DRG; Nav1.8; intrathecal administration; rapamycin

\section{Background}

The most recent surveys show that $12 \%$ of adults have diabetes in China [1], and 16-26\% of diabetic patients experience neuropathic pain [2]. PDN symptoms are intractable because they are often poorly relieved by the currently available analgesics $[3,4]$.

The mammalian target of rapamycin (mTOR) is a key regulator of mRNA translation and protein synthesis, and it is inhibited by rapamycin [5]. mTOR activity is modified in a wide range of pathological states such as cancer and neuronal morphological and functional abnormality associated with tissue damage or neuropathy $[5,6]$. Researchers have suggested that high glucose and insulin resistance were associated with mTOR activation in diabetes [7,8]. mTOR, S6K, 4E-BP are highly present in DRGs, although their active forms are very low under normal conditions $[9,10]$. Evidence has demonstrated that local protein synthesis via the mTOR-depended pathway modulates central peripheral sensitization and is involved in the development and maintenance of chronic pain states [5,10-12]. Recently, researchers proposed that pharmacological or genetic inhibition of mTORC1 contributes to mechanical allodynia via IRS-1(insulin receptor substrate-1)-dependent feedback activation of ERK [13]. However, IR and p-IRS-1 are down-regulated in PDN [14]. It is unknown if repeated administration of rapamycin, inhibiting the activity of mTOR, relieves or induces mechanical hypersensitivity in diabetic rats.

Recent electrophysiological outcomes provide robust evidence that mTOR-mediated local protein synthesis is crucial for neuronal hyperexcitability and synaptic plasticity in the condition of chronic pain [5,10-11]. To date, the exact role of DRG mTOR in neuronal excitability and functional availability has not been examined in a diabetic rat model. Voltage-gated sodium channels (VGSCs) are of paramount importance for nociceptive signal transmission and neuronal hyperexcitability, and the Nav1.8 sodium channels play a key role in VGSCs $[15,16]$. Researchers have revealed that Nav1.8 transcription and expression are reduced in diabetic rats $[17,18]$, and the increased phosphorylation of Nav1.8 mediates an increase in TTXr sodium currents, which underlies diabetic hyperalgesia [18-20]. Therefore, we proposed that DRG mTOR mediates increased phosphorylation of Nav1.8, contributing to PDN. 
In this study, we inhibited DRG mTOR activity by intrathecal administration of rapamycin after the onset of PDN. We used in vitro electrophysiology, behavioral pharmacology, western blotting and RT-PCR to investigate the relationship between the activation of DRG mTOR and the increased phosphorylation of Nav1.8 for the neuronal hyperexcitability and behavior hypersensitivity in STZ-induced diabetic rats.

\section{Materials and methods}

\subsection{Animals}

Male Sprague-Dawley rats (Laboratory Animal Center of Guangdong Province, Guangzhou, China) initially weighing 180-220 g were used and housed in separated cages under standard laboratory conditions, with access to food and water ad libitum. All experimental procedures were approved by the Animal Care Committee of Xuzhou Medical College and were performed in accordance with the guidelines on animal care of the National Institutes of Health and the ethical guidelines [21].

Diabetes was induced by a single intraperitoneal injection of STZ ( $60 \mathrm{mg} / \mathrm{kg}$; Sigma Co., St. Louis, MO, USA) freshly dissolved in citrate buffer ( $\mathrm{pH} 4.5$ ) [22]. Diabetes was confirmed by measuring the blood glucose level 3 days after STZ administration, and rats (54 of 65 rats) with high levels of glucose (>16.7 mmol/L) were used for the diabetic groups; 11 rats were excluded in this study. Age-matched vehicle-injected rats were used as normal controls ( $\mathrm{n}=6$ rats per group for 6 groups). PDN rats were confirmed by examining nociceptive thresholds using von Frey filaments, and in our study, all diabetic rats developed mechanical allodynia at approximately 21 days after STZ administration. All diabetic rats remained relatively healthy, although their growth rate was reduced (body weight gain: 5-10 g/week in the diabetic group versus $50 \mathrm{~g} /$ week in the non-diabetic group).The intrathecal injections were performed on rats at 3 weeks after STZ or vehicle treatment.

\subsection{Intrathecal Catheter Implantation and Drug Administration}

Rats were anesthetized fully using chloral hydrate $(300 \mathrm{mg} / \mathrm{kg})$, and a $1-\mathrm{cm}$ midline incision was made on the dorsal surface. The muscles were retracted to expose the L4-5 vertebrae. Sterile polyethylene tubing (PE-10 catheter) was inserted into the subarachnoid space and advanced $3.5 \mathrm{~cm}$ rostrally at the level of the enlarged spinal cord lumbar segments. The catheter was secured to the paraspinal muscle of the back and then tunneled subcutaneously to exit the dorsal neck region, where it was secured to the skin. The rats were allowed to recover for 4 to 5 days prior to the administration of STZ or vehicle. The position 
of the PE-10 catheter was confirmed by an intrathecal injection of $2 \%$ lidocaine at a volume of $15 \mu \mathrm{L}$, which caused paralysis of both hind limbs. For the rapamycin treatments, the drug was administered intrathecally in $10 \mu \mathrm{L}$ volumes followed by $15 \mathrm{~mL}$ of saline. The rapamycin was dissolved in 4\% DMSO in saline and sonicated prior to i.t. injection. The same volume of 4\% DMSO was administered as a vehicle control.

\subsection{Behavioral Testing}

For assessment of mechanical allodynia, rats were placed in individual Plexiglas compartments with a 20-30 min acclimation period, and the mechanical withdrawal threshold (MWT) was assessed using von Frey filaments (Stoelting, Wood Dale, IL, Chicago, USA) with buckling forces between 0.41 and $15.0 \mathrm{~g}$ according to the up-down method [23]. Mechanical allodynia was defined as the MWT decreasing by more than $50 \%$ from baseline.

\subsection{Western Blotting Analysis}

After the final session of behavioral testing, the rats were deeply anesthetized, and the DRGs were collected. The proteins ( $15 \mathrm{~g})$ extracted from the DRGs were separated on a SDS-PAGE gel and transferred to a PVDF membrane. The membranes were blocked using $5 \%$ nonfat dry milk for 1 hour and incubated overnight at $4{ }^{\circ} \mathrm{C}$ with primary antibodies targeted to phospho-mTOR (p-mTOR, 1:1000, ser-2448, Abcam Inc., Cambridge, MA, UK), mTOR (1:1000, rabbit polyclonal, Cell Signaling Technology, Danvers, MA, USA), phospho-p70S6K (p-S6K, 1:1000, thr-389, Abcam Inc., Cambridge, MA, UK), p70S6K(1:1000, Abcam Inc., Cambridge, MA, UK), Nav1.8((1:1000, Abcam Inc., Cambridge, MA, UK), or phospho-Nav1.8 (p-Nav1.8, S134-12, 1:1000, Abcam Inc., Cambridge, MA, UK). The membranes were then rinsed and incubated with HRP-conjugated and mouse anti-GAPDH (1:10,000; Shanghai Kangcheng Biogo, Shanghai, China) because GAPDH was used as a loading control. The proteins were detected using anti-rabbit or anti-mouse secondary antibodies and visualized using chemiluminescence reagents provided with the Millipore ECL kit (Millipore, NJ, USA) following exposure to hyperfilm (Kodak, Rochester, New York, USA). The intensity of the blots was quantified using densitometry analysis. Densitometry was performed using Image $\mathrm{J}$ software, and the results were normalized to the GAPDH densities of the same sample.

\subsection{Analysis of mRNA expression}


Total RNA was extracted from DRGs using TRIzol (Invitrogen, Carlsbad, CA, USA). In all experiments, $1 \mu \mathrm{g}$ of total RNA was reverse transcribed into cDNA using SuperScript ${ }^{\mathrm{TM}} \mathrm{II}$ RNAse H-reverse transcriptase (Promega, Madison, USA) in a final volume of $12 \mu 1$. Negative controls were performed in which all components were included except for the reverse transcriptase. The relative quantification of mRNA expression was calculated using the comparative $\mathrm{Ct}$ method with the amount of target $=2^{-\Delta \Delta \mathrm{Ct}}[24]$. The values of the target were first normalized to that of the endogenous control ( $\beta$-actin) and then normalized relative to a calibrator. The sequences of the specific primers used for Nav1.8 and $\beta$-actin were as follows:

Nav1.8 sense: 5' GACTCCCGGACAAATCAGAA

Nav1.8 antisense: 5' GGCTGTTTCCTTCCTTAGCA

$\beta$-actin sense: 5' AGGGAAATCGTGCGTGACAT

$\beta$-actin antisense: 5' GAACCGCTCATTGCCGATAG

Real-time PCR was performed using the ABIPRISM ${ }^{\circledR} 7500$ Sequence Detection System (Applied Biosystems, USA). The amplification reactions contained the same amount of RT product (12 $\mu$ l), $2 \times$ Power SYBR Green qPCR SuperMix (Invitrogen, Carlsbad, CA, USA), $300 \mathrm{nM}$ of forward and reverse primers and enough nanopure water to obtain a final volume of $20 \mu \mathrm{l}$. The thermal cycling conditions comprised $2 \mathrm{~min}$ polymerase activation at $95^{\circ} \mathrm{C}$ and 40 cycles of $15 \mathrm{~s}$ at $95^{\circ} \mathrm{C}$ for denaturation and $32 \mathrm{~s}$ at $60^{\circ} \mathrm{C}$ for annealing and extension, followed by a DNA melting curve at $60-95^{\circ} \mathrm{C}$ to determine of amplicon specificity. All experiments were performed in triplicate.

\subsection{Cell culture}

The dorsal vertebral column was opened, and the DRGs were removed and placed into a dish containing Earl's buffer. The DRGs from two animals of each group were combined and incubated for $30-90 \mathrm{~min}$ at $30-35^{\circ} \mathrm{C}$ in oxygenated Earl's buffer containing papain. The cells were dissociated by trituration with a fire-polished Pasteur pipette after rinsing several times with Earl's buffer. The cultures were maintained at $37^{\circ} \mathrm{C}$ in a humidified, atmosphere containing 5\% $\mathrm{CO}_{2}$ in Eagle's minimal essential medium supplemented with $20 \mathrm{mM}$ glucose, $0.5 \mathrm{mM}$ glutamine, $100 \mathrm{U} / \mathrm{ml}$ penicillin, $0.1 \mathrm{mg} / \mathrm{ml}$ streptomycin, and $4 \%$ rat serum.

\subsection{Whole Cell Patch Clamp Recording}

Standard whole-cell patch-clamp recordings from isolated DRGs were performed at room temperature in culture $\left(22-25^{\circ} \mathrm{C}\right)$ using an EPC-10 amplifier and the PULSE program. The 
electrodes (2-5 M) were pulled using standard-wall glass pipettes (Institute of Neuroscience, Shanghai, China) using a horizontal puller PB-7 (Narishige, Japan) and were filled with the following reagents (in $\mathrm{mM}$ ): $1 \mathrm{CaCl}_{2}, 120 \mathrm{CsCl}, 5 \mathrm{MgCl}_{2}, 11 \mathrm{EGTA}, 10 \mathrm{HEPES}, 11$ glucose, $5 \mathrm{Na}_{2} \mathrm{ATP}$, the $\mathrm{pH}$ was adjusted to 7.3 using $\mathrm{CsOH}$, and the osmolarity was adjusted to 285 mOsm. The pipette solutions were filtered using $0.2 \mu \mathrm{m}$ filters immediately before use. The bath solution used to record currents contained the following reagents (in $\mathrm{mM}$ ): $30 \mathrm{NaCl}, 110$ choline-Cl, $1 \mathrm{MgCl}_{2}, 0.1 \mathrm{CaCl}_{2}, 5.4 \mathrm{KCl}, 0.33 \mathrm{NaH}_{2} \mathrm{PO}_{4}$, and $10 \mathrm{HEPES}$. The $\mathrm{pH}$ was adjusted to 7.4 using $\mathrm{NaOH}$, and the osmolarity was $325 \mathrm{mOsm}$. A reduced extracellular $\mathrm{Na}^{+}$ was required to reduce the magnitude of sodium currents to improve the fidelity of the voltage clamp [25]. Under these recorded conditions, the estimated Nernst reversal potential for $\mathrm{Na}^{+}$ was $-30 \mathrm{mV}$. In some experiments, action potentials were recorded in the current clamp mode using methods described previously [26]. TTX-S and TTX-R currents were isolated pharmacologically by adding TTX (200 nM, Sigma, Shanghai, China) to the bath solution. The total current was evoked from a holding potential of $-120 \mathrm{mV}$ to the test pulses, which ranged from -90 to $+30 \mathrm{mV}$, in increments of $5 \mathrm{mV}$ at a frequency of $0.2 \mathrm{~Hz}$, and TTX (200 nM) was then added to the bath solution to record TTX-resistant currents. Recording data were acquired using a Digital / analog converter PCI-16 board (HEKA, German) and were stored on a computer using Pulse + Pulsefit software (version 8.31).

Activation and steady-state inactivation data were fitted using a Boltzmann equation of the form, $G=G_{\max } /\left(1+\exp \left(V_{50}-V_{m}\right) / k\right)$. Here, $G=I /\left(V_{m}-V_{\text {res }}\right)$, where $V_{m}$ is the potential at which current is evoked, and Versa is the reversal potential for the current determined by extrapolating the linear portion of the I-V curve through 0 current, $G_{\max }=$ the calculated maximal conductance, $\mathrm{V}_{50}=$ the potential of half activation or inactivation, and $\mathrm{k}=$ the slope factor.

\subsection{Statistics}

The data analyses and statistical comparisons were performed using SPSS 15.0 (SPSS Inc., USA). All data are presented as the mean \pm SD. When the normality and homogeneity of variance assumptions were satisfied, differences in the changes of values between groups were tested using one-way ANOVAs followed by Student-Newman-Keuls tests or by using Student's t-tests if only two groups were analyzed. If after the logarithmic transformation the data did not show homogeneity of variance, the equivalent non-parametric tests were employed. For data from the behavioral tests, a repeated measure of general linear model 
ANOVAs was employed to compare the data from various testing days. The criterion for significance was set at $P<0.05$.

\section{Results}

\subsection{Assessment of allodynia}

The diabetic rats exhibited hypersensitivity to innocuous mechanical stimuli of the hind paw as early as 9 days after the injection of STZ, and robust significant mechanical allodynia appeared on day 21 after the STZ injection and was maintained until at least 4 weeks after the endpoint of the experiment (Figure.1). This procedure has been found to produce long-lasting hypersensitivity to noxious mechanical or heat stimuli for at least 8 weeks after a STZ injection [3], which perhaps reflects the longevity of the chronic symptoms of diabetes in this animal model.

\subsection{Activation of the mTOR pathway and increases in the phosphorylation of} Nav1.8 in DRGs are accompanied by a decrease in the mechanical withdrawal threshold in STZ-induced diabetic rats

We utilized STZ-induced diabetic rats to assess mTOR and changes in its downstream signaling partners in DRGs. The majority of rats developed hyperglycemia after an injection of STZ. The diabetic rats exhibited significant mechanical allodynia in response to applications of von Frey filaments at three weeks after the injection of STZ, and the MWT showed a slight increase at four weeks (Figure.1). We observed that p-mTOR and total mTOR greatly increased following a dramatic escalation in the expression of p-Nav1.8 prior to the marked reduction in MWT (Figure.1 and Figure.2). A significant increase in mTOR and p-Nav1.8 was observed at two weeks after the STZ injection $(p<0.05)$, and p-mTOR (Ser-2448) began to increase during the first week $(p<0.05)$; Nav1.8 showed a slight increase only during the first week (Figure 3).

\subsection{Activation of the mTOR pathway promotes the phosphorylation of Nav1.8} and contributes to the behavioral hypersensitivity, and intrathecal administration of rapamycin abolishes these changes in STZ-induced diabetic rats

We investigated how mTOR activation induces Nav1.8 posttranslational modifications and neuronal hyperexcitability in DRGs following an injection of STZ. The Nav1.8 protein and mRNA levels were measured in DRG samples from diabetic rats and were compared with 
normal controls. Immunoreactivity for anti-phospho Nav1.8 was increased significantly, and a slight decrease was observed in the Nav1.8 protein and mRNA levels (Figure.4). The intrathecal administration of rapamycin (i.t.; $1 \mu \mathrm{g}, 3 \mu \mathrm{g}$, or $10 \mu \mathrm{g} /$ day) for 7 days markedly inhibited the activation of mTOR (phospho-S6K is a specific phosphorylated target of mTOR and is widely used in monitoring its activation [24]), decreased the expression of total protein and phosphorylation of Nav1.8 and ameliorated hyperalgesia in a dose-dependent manner (Figure.3 and Figure.4). However, an increased dose did not inhibit Nav1.8 transcription significantly (Figure.4D).

\subsection{Activation of the mTOR pathway is associated with TTX-R sodium channel availability and increased current, and intrathecal rapamycin inhibited these changes in DRGs}

We used electrophysiological techniques to assess the mTOR regulation of amplitude and properties of the TTX-R I $\mathrm{I}_{\mathrm{Na}}$ in cultured DRG neurons of diabetic rats. To record TTX-R sodium currents $\left(\mathrm{I}_{\mathrm{Na}}\right)$, standard whole-cell patch-clamp recordings were taken from isolated neurons of group C (normal control), group D (diabetes-vehicle) and group $\mathrm{R}$ (diabetes-rapamycin, $10 \mu \mathrm{g}$ ) rats. The cells were voltage clamped at $-90 \mathrm{mV}$ with wave widths of $40 \mathrm{~ms}$, and currents were elicited by graded increases in the voltage from- $80 \mathrm{mV}$ in the presence of $200 \mathrm{nM}$ TTX (in the bath solution). The peak currents were significantly higher in the neurons from group D (Figure 5A). Original current traces of TTX-R $\mathrm{I}_{\mathrm{Na}}$ were recorded in DRGs prepared from a diabetic rat at 4 weeks after the onset of diabetes compared with an age-matched control, and the amplitude of outward $\mathrm{I}_{\mathrm{Na}}$ was significantly larger in the neurons from the diabetic rats compared with the controls; rapamycin (i.t., $10 \mu \mathrm{g} /$ day for 7 days) reduced this increase in amplitude (Figure.5A). As shown in Figure.5B, the current-voltage relationship was calculated as the ratio of each membrane maximum current and capacitance and was shifted in the hyperpolarizing direction for DRGs isolated from diabetic rats compared with those from isolated normal control rats. The peak current trigger voltage was $-10 \mathrm{mV}$ in the neurons from group $\mathrm{C}$, whereas it was $-20 \mathrm{mV}$ in the neurons from group $\mathrm{D}$ and group $\mathrm{R}$. The mean peak current density in the neurons from group $\mathrm{R}$ was higher than in the group $\mathrm{C}$ neurons $(-40.8 \pm 1.3 \mathrm{pA} / \mathrm{pF} v s-35.2 \pm 1.7 \mathrm{pA} / \mathrm{pF}, p<0.05, \mathrm{n}=15)$ but was significantly lower than in the group D neurons $(-40.8 \pm 1.3 \mathrm{pA} / \mathrm{pF} v s-46.3 \pm 1.9 \mathrm{pA} / \mathrm{pF}, p$ $<0.05, \mathrm{n}=15)$.

The kinetics of activation were evaluated by curve fitting the rising phase of the conductance using a single exponential function Boltzmann equation (Figure.5C).The currents $\left(\mathrm{I}_{\mathrm{Na}}\right)$ under different test voltages were calculated and standardized as the $\mathrm{I}_{\mathrm{Na}} / \mathrm{I}_{\mathrm{Na}}$ 
$(\max )$ of each current. We applied the Boltzmann equation, $\mathrm{I}_{\mathrm{Na}} / \mathrm{I}_{\mathrm{Na}}(\max )=1 /\{1+\exp$ $[(\mathrm{V} 1 / 2-\mathrm{V}) / \mathrm{k}]\}$, and fitted a curve to determine the half-activation voltage $\mathrm{V} 1 / 2$ of the activation curve slope factor $\mathrm{k}$. The midpoint of the voltage-dependence of activation was $-19.3 \pm 1.3 \mathrm{mV}$ in cells from the rats in group $\mathrm{R}$ and was $-21.5 \pm 1.2 \mathrm{mV}$ in the neurons from group $\mathrm{D}$. These values were significantly higher in the neurons from group $\mathrm{R}$ and group $\mathrm{D}$ than in the neurons from group $\mathrm{C}(-16.6 \pm 1.4 \mathrm{mV} ; p<0.01)$, and the values were higher in group $\mathrm{R}$ than in group $\mathrm{D}(p<0.05)$. Moreover, the activation curve slope $\mathrm{k}$ was $7.09 \pm 0.20$ in group C, $8.14 \pm 0.17$ in group $\mathrm{D}$ and $8.47 \pm 0.15$ in the neurons from group $\mathrm{R}$. The difference between groups was statistically significant $(p<0.05)$. The voltage dependence of steady-state inactivation was similarly calculated using the Boltzmann equation. The midpoints of steady-state inactivation were $-22.7 \pm 1.8 \mathrm{mV}$ in the neurons from group $\mathrm{C}$ rats and was less negative than in the neurons from group $\mathrm{D}(-33.6 \pm 2.1 \mathrm{mV})$ or group $\mathrm{R}(-28.5 \pm$ $1.9 \mathrm{mV} ; p<0.05)$. The difference between these values in the neurons of group $\mathrm{D}$ and group $\mathrm{R}$ was statistically significant $(p<0.05)$ (Figure.5D). Therefore, the steady-state inactivation of TTX-R currents was negatively shifted in the DRG neurons from group D and group R compared with the neurons from group C (Figure.5D).

\section{Discussion}

Changes in the expression, trafficking, and function of the tetrodotoxin-resistant (TTX-R) sodium channel have been proposed to underlie the neuronal hyperexcitability observed in the pain-like phenotype of neuropathic animal models [26]. Our data also show that the up-regulation of the phosphorylation of Nav1.8 mediates hyperalgesia in STZ-induced rats, which is consistent with the reports of Hong and Bierhauss.

Increasing evidence suggests that the mTOR signaling pathway, as an important modulator of translational control, is involved in persistent pain [5,10,11]. Herein, we investigated the molecular changes of DRGs in diabetic rats. Our results show that DRG mTOR is an important regulator of peripheral sensitization, inflammation and neuropathy, and an intrathecal (i.t.) injection of rapamycin inhibited the activity of DRG mTOR and relieved hyperalgesia [10]. Furthermore, increasing evidence shows that the activation of the mTOR pathway mediates neuronal plasticity and hyperexcitability and modulates the transmission of nociceptive information in chronic pain states [5,10,27]. In our study, DRG mTOR was highly increased and activated, followed by a dramatic increase in the expression level of p-Nav1.8, all prior to the marked decrease in the mechanical withdrawal threshold. Furthermore, the intrathecal administration of rapamycin (i.t.; $1 \mu \mathrm{g}, 3 \mu \mathrm{g}$, or $10 \mu \mathrm{g}$ /day for 7 
days) inhibited the function of p-mTOR, effectively suppressed the phosphorylation of Nav1.8 and relieved hyperalgesia in STZ-induced rats. The TTX-R sodium current density $\left(\mathrm{I}_{\mathrm{Na}}\right)$ increased significantly in DRGs isolated from diabetic rats, and the voltage-dependent activation and steady-state inactivation curves for these currents were shifted negatively (Figure.5). The rapamycin (i.t., $3 \mu \mathrm{g} /$ day for 7 days) treatment suppressed the phosphorylation of Nav1.8, reduced the current density and elevated the MWT. We propose that mTOR modulates the phosphorylation of Nav1.8 and current density associated with neuronal excitability.

Studies have confirmed that the intrathecal administration of rapamycin blocks the activities of mTOR in the dorsal horn $[9,11,28]$ and dorsal roots at $2 \mathrm{~h}$ after the intrathecal injection, but it does not block the DRG activity [28]. However, these studies did not determine the effect of an intrathecal injection of rapamycin on the activity of DRG mTOR under chronic conditions. Our results show that the intrathecal administration of rapamycin has an effect on DRG mTOR, which is reported by other researchers [29], and a possible explanation for this phenomenon is that the duration of our treatment is longer than the duration of other treatments, and the drug targets DRGs at a mean of axoplasmic transport; however, we are unable to rule out that the intrathecal administration of rapamycin might attenuate hypersensitivity through a combined peripheral (DRG) and central (dorsal horn) action. Recently, research finds that chronic treatment with rapamycin induces mechanical allodynia via IRS-1-dependent feedback activation of ERK [13], although our results show that a repeated intrathecal injection ( $1 \mu \mathrm{g}, 3 \mu \mathrm{g}$ or $10 \mu \mathrm{g} /$ day for 7 days) of rapamycin does not induce mechanical hyperalgesia in a PDN model. A possible explanation for this finding is that IR and p-IRS-1 are down-regulated in painful diabetic neuropathy [14], and the chronic administration of mTORC1 inhibition could not induce mechanical hypersensitivity.

In our study, we use three doses (i.t., $1 \mu \mathrm{g}, 3 \mu \mathrm{g}$ or $10 \mu \mathrm{g} /$ day for 7 days) to treat painful diabetic neuropathy and to observe the changes in biological molecules because the dosage and duration have been proven to be safe and effective in rats with intractable and persistent hyperalgesia [30]. PDN and cancer-related pain have been shown to be intractable and refractory to currently available treatment. Norsted Gregory and his collogues demonstrated that an injection of rapamycin (0.1-3 $\mu \mathrm{g})$ led to a dose-dependent decrease in carrageenan-induced thermal hyperalgesia and a reduction in mechanical allodynia [11]. However, in our preliminary experiments, we found that a dosage less than $3 \mu \mathrm{g}$ /day for 7 days was not effective in PDN rats. Considering the efficacy and safety, we adopted a dose of $3 \mu \mathrm{g}$, which had an effective pain relief rate and a smaller effect on normal function 
(unpublished data), to use in the electrophysiological studies. However, our study might have some potential limitations. First, crystallographic and site-directed mutagenesis studies on these voltage-gated sodium channels (VGSCs) are unavailable, and the exact molecular link between mTOR and changes in channel function remain unknown. However, the biochemical and functional data presented herein support the hypothesis that mTOR modulates the phosphorylation of Nav1.8 associated with PDN. Second, there were no control rats treated with rapamycin only in our study because research has confirmed that no significant difference is caused by the doses of rapamycin that were used in our study [10,11]. Moreover, other groups have demonstrated that the level of serine/threonine phosphorylation of the Nav1.8 protein or modifications of the arginine residue(s) within the DIII-DIV linker of Nav1.8 (which comprises its inactivation gate) were increased and might be involved in the increase in TTX-R sodium current density in DRGs from diabetic rats $[17,20]$. Therefore, we are unable to rule out that these sites contributed to alterations in the modulating the properties of Nav1.8; however, these possibilities would be investigated in our future studies. In our work, we did not exclude that i.t. rapamycin might also mediate the inhibition of spinal mTOR activity.

\section{Conclusion}

DRG mTOR partly modulated the phosphorylation of Nav1.8 and its functional properties contributing to hyperalgesia in diabetic rats, and intrathecal (it) injection of rapamycin dose-dependently inhibited the activities of mTOR and the phosphorylation of Nav1.8 and attenuated behavioral hypersensitivity in STZ-induced diabetic rats. These results suggest that rapamycin is a potential therapeutic intervention for clinical PDN.

\section{Acknowledgments}

This study was supported by the Guangdong National Science Foundation Grant 1414050003575. The authors declare that they have no competing interests.

\section{References}

[1] Yu Xu, Limin Wang, Jiang He, MD, et al. Prevalence and Control of Diabetes in Chinese Adults. JAMA. 2013, 310(9):948-959.

[2] Abbott CA, Malik RA, van Ross ER, Kulkarni J, Boulton AJ. Prevalence and characteristics of painful diabetic neuropathy in a large community-based diabetic population in the U.K. Diabetes Care, 2011, 34(10):2220-2224. 
[3] Jensen TS, Backonja MM, Hernández Jiménez S, Tesfaye S, Valensi P, Ziegler D, New perspectives on the management of diabetic peripheral neuropathic pain. Diab Vasc Dis Res 3 (2006) 108-119.

[4] Chen SR, Chen H, Yuan WX, Pan HL, 2007. Increased Pre- and Postsynaptic a2-Adrenoceptor Activity in the Spinal Dorsal Horn in Painful Diabetic Neuropathy. J Pharmacol Exp Ther 337 (1), 285-92.

[5] Asante CO, Wallace VC, Dickenson AH, 2010. Mammalian Target of Rapamycin Signaling in the Spinal Cord Is Required for Neuronal Plasticity and Behavioral Hypersensitivity Associated With Neuropathy in the Rat. The Journal of Pain 11 (12), 1356-1367.

[6] Ma XM, Blenis J, Molecular mechanisms of mTOR-mediated translational control. Nat Rev Mol Cell Biol 10 (2009) 307-318.

[7] Velagapudi C, Bhandari BS, Abboud-Werner S, Simone S, Abboud HE, Habib SL, The Tuberin/mTOR Pathway Promotes Apoptosis of Tubular Epithelial Cells in Diabetes. J Am Soc Nephrol 22 (2011) 262-273.

[8] Amanda E. Brandon, Andrew J. Hoy, Lauren E. Wright, Nigel Turner, Bronwyn D. Hegarty, Tristan J. Iseli, X. Julia Xu, Gregory J. Cooney, Asish K. Saha, Neil B. Ruderman, Edward W. Kraegen, The evolution of insulin resistance in muscle of the glucose infused rat. Arch Biochem Biophys 509 (2011) 133-141.

[9] Xu JT, Zhao X, Yaster M, Tao YX, Expression and distribution of mTOR, p70S6K, 4E-BP1, and their phosphorylated counterparts in rat dorsal root ganglion and spinal cord dorsal horn. Brain Res 1336 (2010) 46-57.

[10] Asante CO, Wallace VC, Dickenson AH, Formalin-induced behavioural hypersensitivity and neuronal hyperexcitability are mediated by rapid protein synthesis at the spinal level. Mol Pain 5 (2009) 27.

[11] Norsted Gregory E, Codeluppi S, Gregory JA, Steinauer J, Svensson CI: Mammalian target of rapamycin in spinal cord neurons mediates hypersensitivity induced by peripheral inflammation. Neuroscience 169 (2010) 1392-1402

[12]Jiménez-Díaz L, Géranton SM, Passmore GM, Leith JL, Fisher AS, Berliocchi L, Sivasubramaniam AK, Sheasby A, Lumb BM, Hunt SP, Local Translation in Primary Afferent Fibers Regulates Nociception. PLoS One 3 (2008) e1961.

[13] Melemedjian OK, Khoutorsky A, Sorge RE, Yan J, Asiedu MN, Valdez A, Ghosh S, Dussor G, Mogil JS, Sonenberg N,Price TJ. mTORC1 inhibition induces pain via IRS-1-dependent feedback activation of ERK. Pain 154 (2013) 1080-1091. 
[14] Kou ZZ, Li CY, Tang J, Hu JC, Qu J, Liao YH, Wu SX, Li H, Li YQ, Down-regulation of insulin signaling is involved in painful diabetic neuropathy in type 2 diabetes. Pain Physician 16(2013) E71-83

[15] Renganathan M, Cummins TR, Waxman SG, Contribution of $\mathrm{Na}(\mathrm{v}) 1.8$ sodium channels to action potential electrogeness in DRGs. J Neurophysiol 86 (2001) 629-640.

[16] Liu C, Li Q, Su Y, Bao L, Prostaglandin E2 Promotes Na1.8 Trafficking via Its Intracellular RRR Motif through the Protein Kinase A Pathway. Traffic 11(2010.) 405-417.

[17] Craner MJ, Klein JP, Renganathan M, Black JA, Waxman SG, Changes of sodium channel expression in experimental painful diabetic neuropathy. Ann Neurol 52(2002) 786-792.

[18] Hong S, Morrow TJ, Paulson PE, Isom LL, Wiley JW, Early painful diabetic neuropathy is associated with differential changes in tetrodotoxin-sensitive and -resistant sodium channels in dorsal root ganglion neurons in the rat. J Biol Chem 279 (2004) 29341-29350.

[19] Shah BS, Gonzalez MI, Bramwell S, Pinnock RD, Lee K, Dixon AK, Beta3, a novel auxiliary subunit for the voltage gated sodium channel is upregulated in sensory neurones following streptozocin induced diabetic neuropathy in rat. Neurosci Lett 309 (2001) 1-4.

[20] Bierhaus A, Fleming T, Stoyanov S, Leffler A, Babes A, Neacsu C, Sauer SK, Eberhardt M, Schnölzer M, Lasitschka F, Methylglyoxal modification of Nav1.8 facilitates nociceptive neuron firing and causes hyperalgesia in diabetic neuropathy. Nat Med 18 (2012) 926-933.

[21] Zimmermann M, Ethical guidelines for investigations of experimental pain in conscious animals. Pain 16 (1983) 109-110.

[22] Zhao WC, Zhang B, Liao MJ, Zhang WX, He WY, Wang HB, Yang CX, Curcumin ameliorated diabetic neuropathy partially by inhibition of NADPH oxidase mediating oxidative stress in the spinal cord. Neurosci Lett 560 (2014) 81-85.

[23] Chaplan SR, Bach FW, Pogrel JW, Chung JM, Yaksh TL, 1994. Quantitative assessment of tactile allodynia in the rat paw. J Neurosci Methods, 53(1), 55-63.

[24] Livak KJ, Schmittgen TD, Analysis of relative gene expression data using real-time quantitative PCR and the 2(-Delta Delta C(T)) method. Methods 25(2001), 402-408.

[25] McGaraughty S, Chu KL, Scanio MJ, et al. A selective Nav1.8 sodium channel blocker, A-803467, attenuates spinal neuronal activity in neuropathic rats. J Pharmacol Exp Ther 2008, 324(3):1204-1211. 
[26] Renganathan M, Cummins TR, Waxman SG. Contribution of Na(v)1.8 sodium channels to action potential electrogeness in DRG neurons. J Neurophysiol 2001, 86(2):629-640.

[27] Weragoda RM, Ferrer E, Walters ET, Memory-like alterations in Aplysia axons after nerve injury or localized depolarization. J Neurosci 24(2004) 10393-10401.

[28] Géranton SM, Jiménez-Diaz L, Torsney C, Tochiki KK, Stuart SA, Leith JL, Lumb BM, Hunt SP: A rapamycin-sensitive signaling pathway is essential for the full expression of persistent pain states. J Neurosci 29(2009) 15017-15027.

[29] Ghelardini C, Menicacci C, Cerretani D, Bianchi E, Spinal administration of mGluR5 antagonist prevents the onset of bortezomibinduced neuropathic pain in rat.

Neuropharmacology 86 (2014) 294-300.

[30] Shih MH, Kao SC, Wang W, Yaster M, Tao YX: Spinal Cord NMDA

Receptor-Mediated Activation of Mammalian Target of Rapamycin Is Required for the Development and Maintenance of Bone Cancer-Induced Pain Hypersensitivities in Rats. The Journal of Pain 2012, 13 (4):338-349.

\section{Figure legends}

Figure.1. This graph shows the MWT at different time points in grams $(\mathrm{g})$ prior to $(\mathrm{t}=0$ day) and after intraperitoneal (i.p.) injection of STZ. The data are shown as the mean \pm SD, $\mathrm{n}=6$ rats per group. $*$ denotes $p<0.05$ compared with normal control. \# denotes $p<0.05$ compared with baseline.

Figure.2. These bar graphs display the percentage change from normal controls ( $t=0$ day) for (A) mTOR, (B) phospho(p)-mTOR, (C) Nav1.8 and (D) p-Nav1.8 in DRGs at different time points following STZ-induced diabetes in rats. Representative Western blots of mTOR, p-mTOR, Nav1.8 and p-Nav1.8 are shown in the front of the bar graphs. The data are shown as the percentage change from normal control and are expressed as the means $\pm \mathrm{SD}, \mathrm{n}=6$ rats per group. * denotes $p<0.05$ compared with normal controls.

Figure.3. Variation in the WWTs of rats. The data are shown as the mean $\pm \mathrm{SD}, \mathrm{n}=6$ rats per group. ${ }^{*}$ denotes $p<0.05$ compared with normal control. \# denotes $p<0.05$ compared with diabetic vehicle treatment. $\$$ denotes $p<0.05$ compared with diabetic rats treated with $1 \mu \mathrm{g}$ rapamycin. \& denotes $p<0.05$ compared with diabetic rats treated with $3 \mu \mathrm{g}$ rapamycin. 
Figure.4. The influence of repeated intrathecal administration of rapamycin on DRG protein expression of $\mathrm{p}-\mathrm{S} 6 \mathrm{~K}, \mathrm{Nav1.8}$, p-Nav1.8 protein and mRNA expression of Nav1.8 mRNA. A-C, effect of rapamycin on p-S6K (A) and Nav1.8 (B) protein expression and phosphorylation levels (C). D, The panel depicts a representative RT-PCR gel that shows the relative gene expression of Nav1.8 in DRGs. The abbreviations for the groups of normal control, diabetic-vehicle (4\% DMSO), diabetes-rapamycin $1 \mu \mathrm{g}, 3 \mu \mathrm{g}$ and $10 \mu \mathrm{g}$ are shown as $\mathrm{C}, \mathrm{D}, \mathrm{R} 1, \mathrm{R} 3$ and R10, respectively. Compared with group $\mathrm{C},{ }^{*} p<0.05$; compared with group $\mathrm{D},{ }^{\#} p<0.05$; compared with group $\mathrm{R} 1,{ }^{\$} p<0.05$; compared with group $\mathrm{R} 2,{ }^{\&} p<0.05$.

Figure.5. Changes in amplitude and properties of TTX-R $\mathrm{I}_{\mathrm{Na}}$ in cultured DRGs from diabetic or normal control rats. A, Typical traces of TTX-R $\mathrm{I}_{\mathrm{Na}}$ conducted in voltage-clamp mode in cultured neurons from normal control rats (top), diabetes + vehicle rats (middle) and diabetes-rapamycin rats (10 $\mu \mathrm{g}$; bottom). B, Line graph showing the current voltage relationship using current density as the indicator for $\mathrm{I}_{\mathrm{Na}}$ in DRGs from normal control rats and diabetic rats. $\mathbf{C}$, Normalized current-voltage curves of $\mathrm{I}_{\mathrm{Na}}$ activation. $\mathbf{D}$, Normalized current-voltage curves of steady state inactivation. 


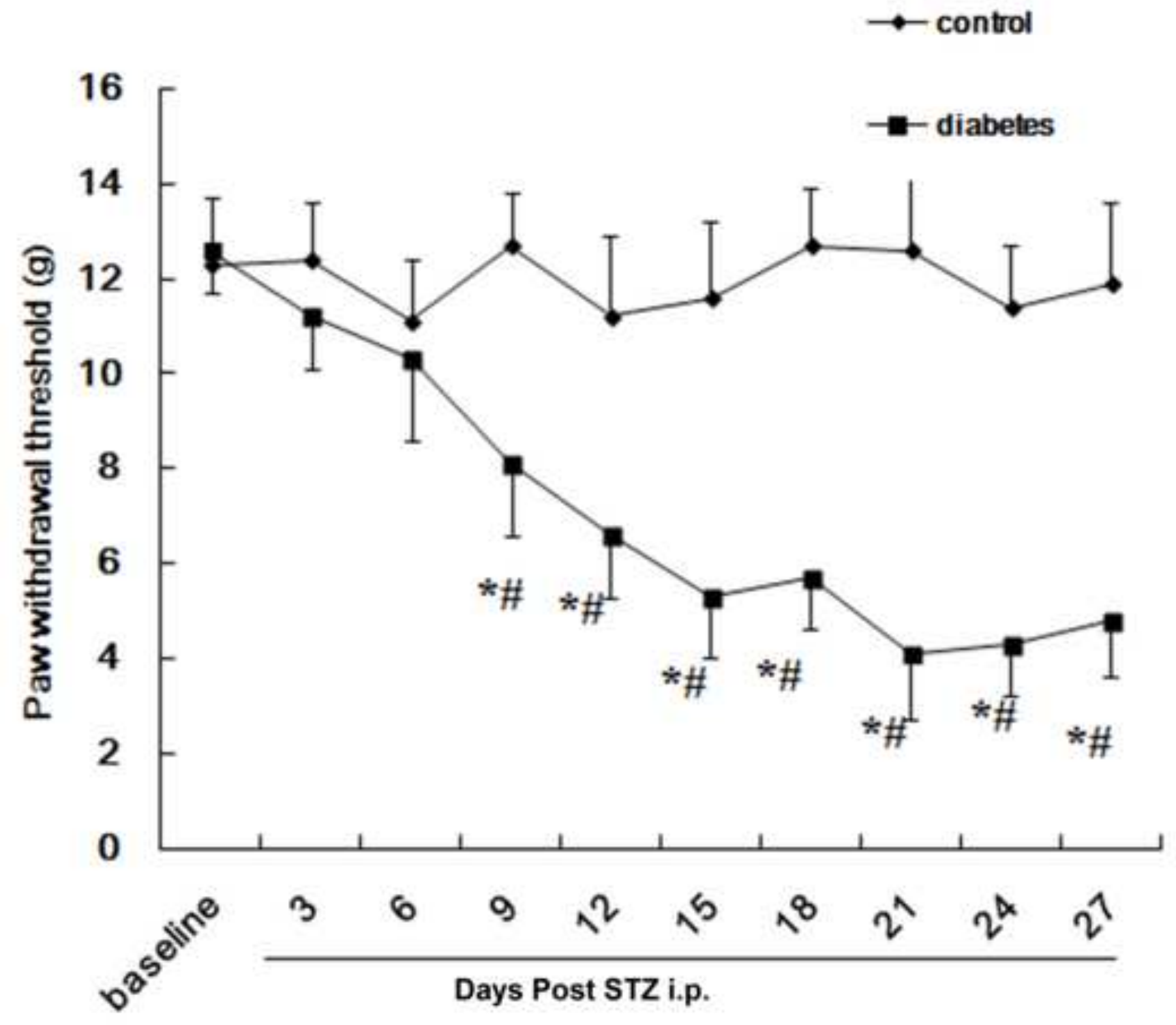



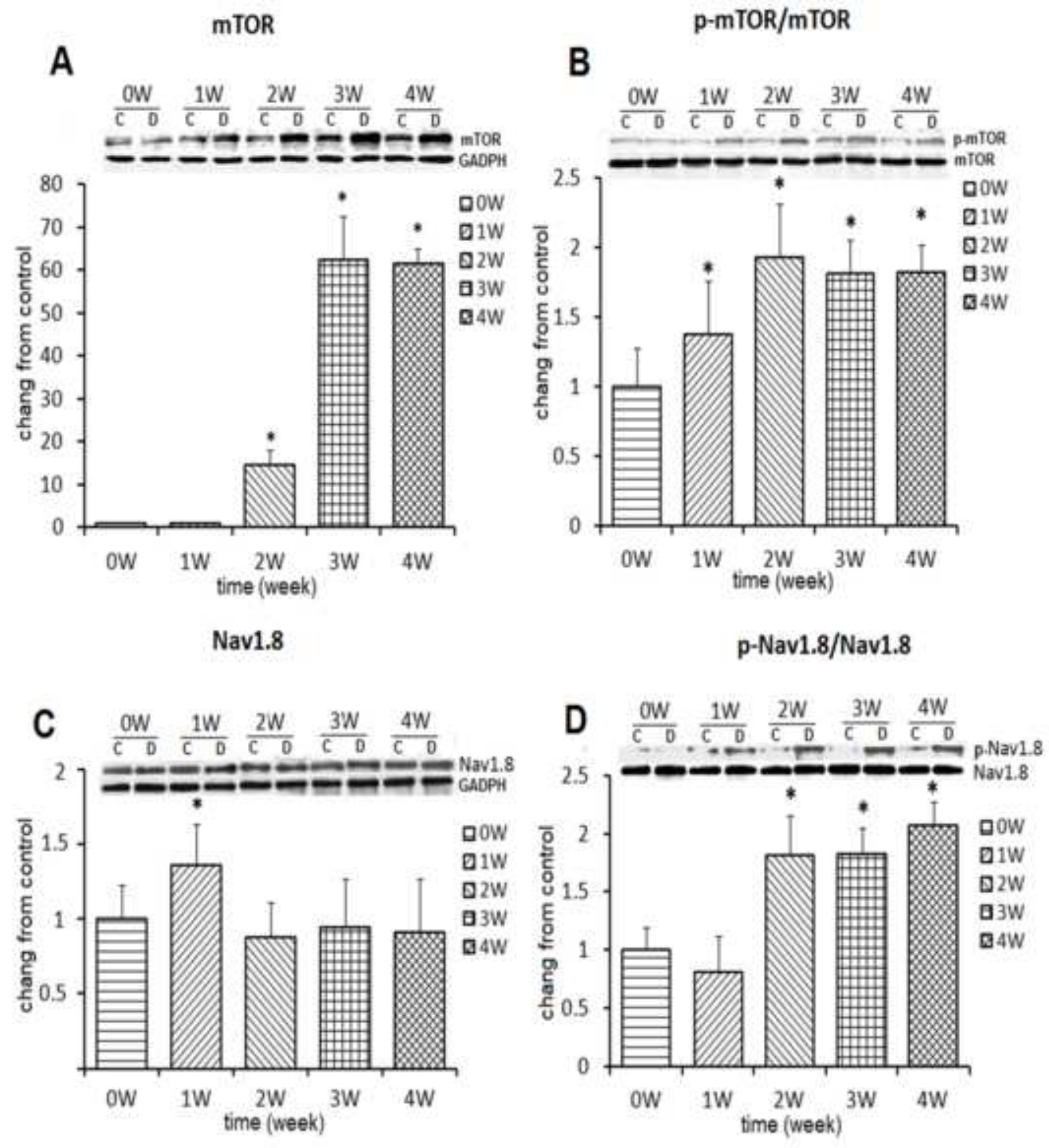

Figure.2 


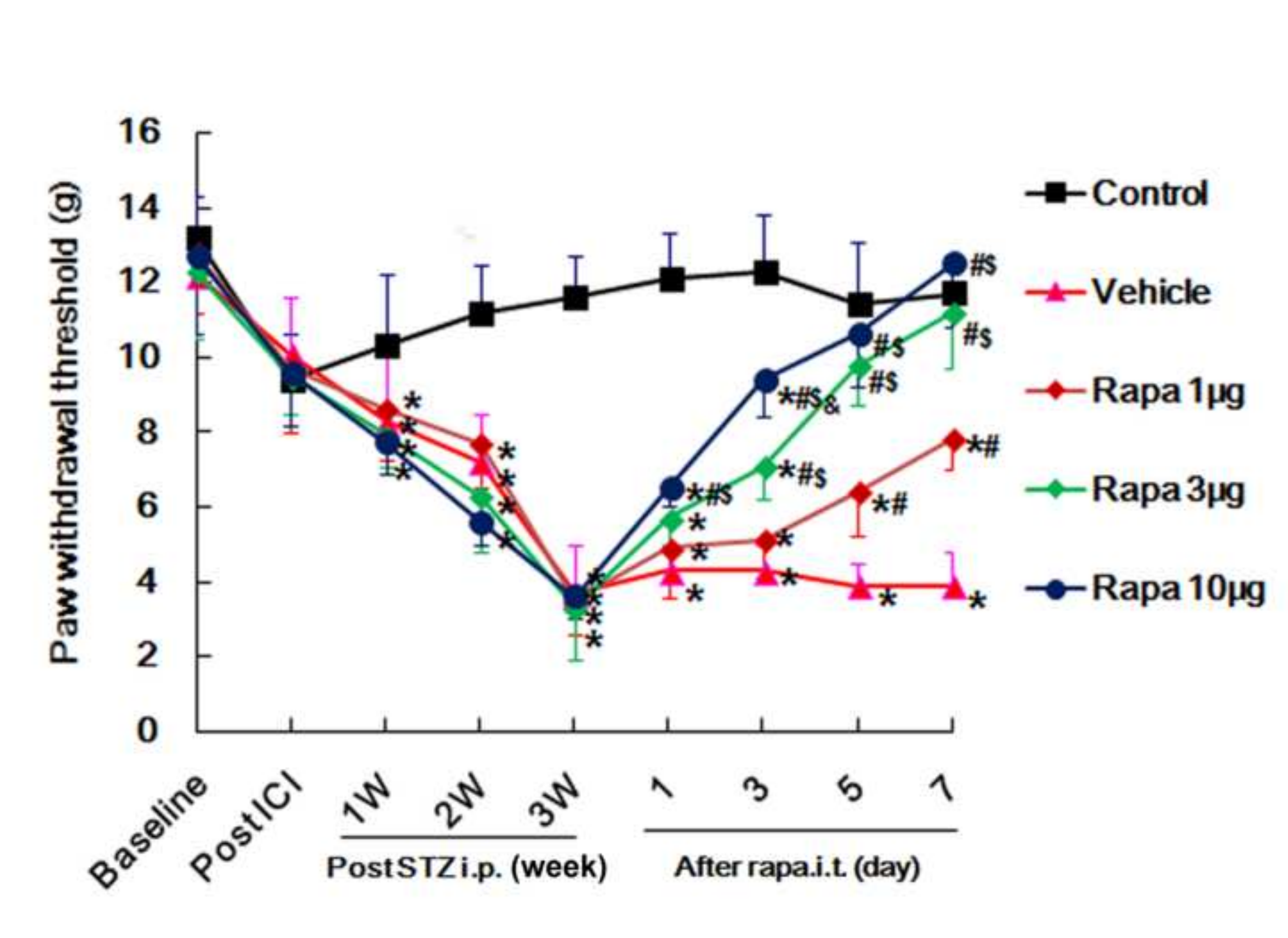

.

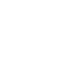

.

(1)

.

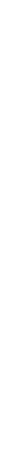



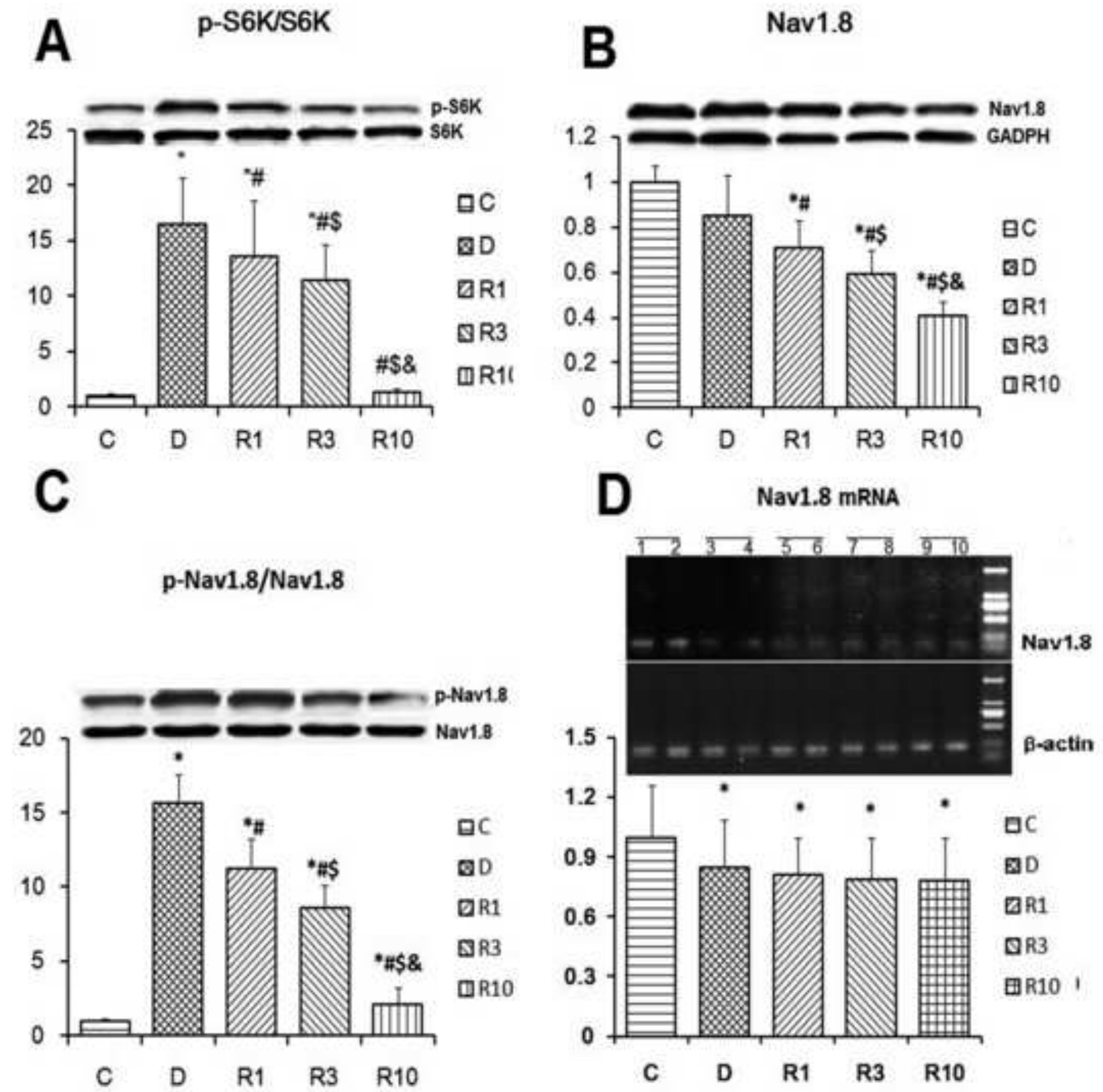

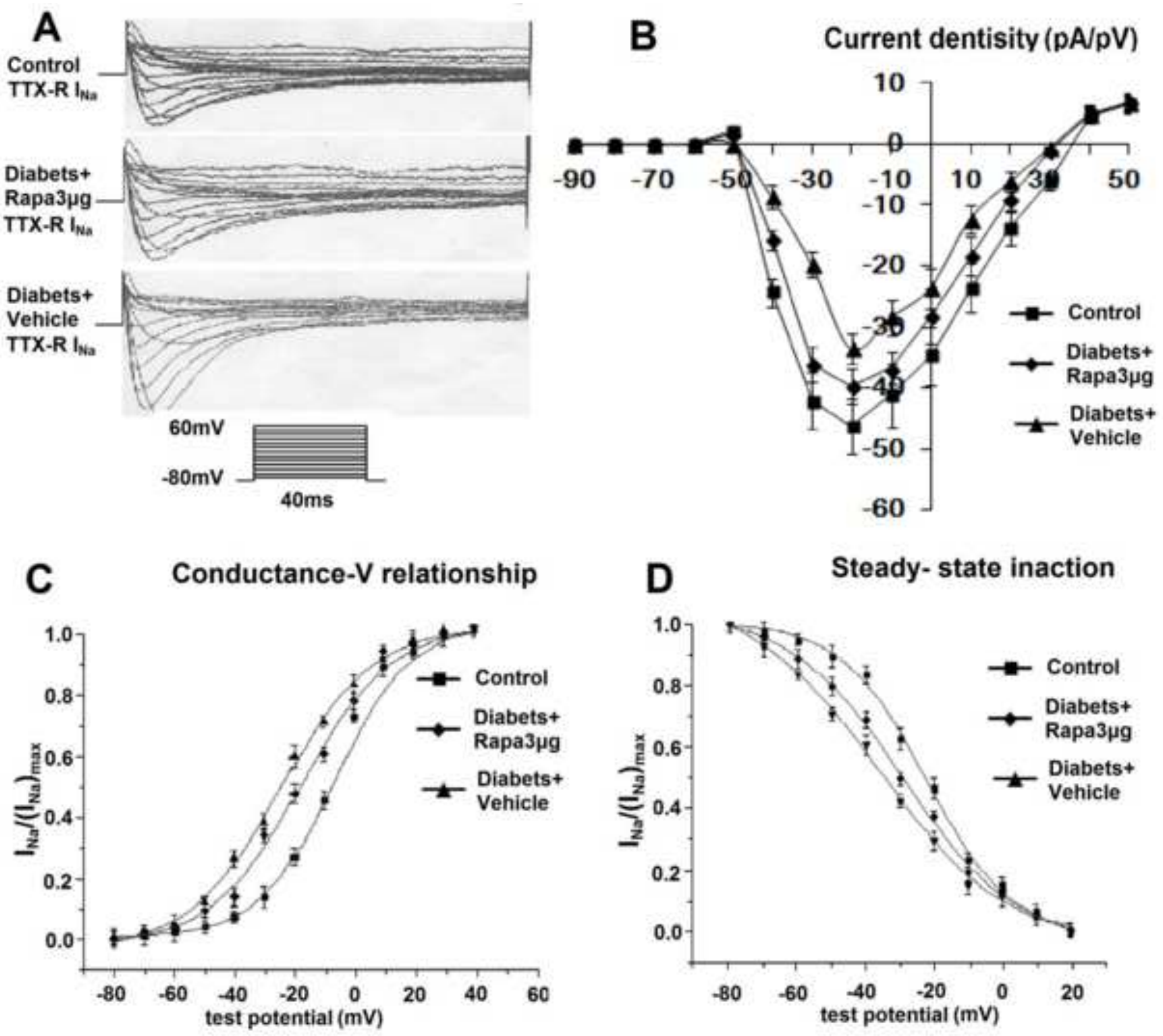